\title{
ON COMMUTATIVITY OF THE SECOND-ORDER CROSS PARTIAL DERIVATIVES
}

\author{
SOLOMON MARCUS
}

In $1956 \mathrm{~J}$. Geffroy has given the following theorem:

Let $f$ be a real function defined at each point of a plane domain $G$. If $f_{x y}^{\prime \prime}=\left(f_{x}^{\prime}\right)_{y}^{\prime}$ and $f_{y x}^{\prime \prime}=\left(f_{y}^{\prime}\right)_{x}^{\prime}$ exist and are finite in $G$, and $f_{x y}^{\prime \prime}$ is bounded in $G$ then $f_{x y}^{\prime \prime}=f_{y x}^{\prime \prime}$ in $G$ if and only if $f_{x y}^{\prime \prime}$, considered as a function of $x$, is a derivative function [2].

To establish this theorem, J. Geffroy uses as a main tool Lebesgue's integral. But it is known that there are derivative functions which are not integrable in Lebesgue sense. It is more natural to use in this problem the Denjoy-Perron integral, which permits us to integrate each finite derivative function. But some general theorems concerning the Denjoy-Perron integral are too complicated to be used for practical purposes. The passage to limit under the integral sign, which is essential in Geffroy's proof, is a very difficult problem for the Denjoy-Perron integral. By modifications in Geffroy's proof, I have replaced the boundedness of $f_{x y}^{\prime \prime}$ by the continuity of $f_{x}^{\prime}$ with respect to $x$. I think that these conditions are not essential, being required only by the method of proof. I make the following

CONJECTURE. If $f_{x y}^{\prime \prime}$ and $f_{y x}^{\prime \prime}$ exist and are finite in $G$, then $f_{x y}^{\prime \prime}=f_{y x}^{\prime \prime}$ in $G$ if and only if $f_{x y}^{\prime \prime}$ is, for each $y, a$ derivative function with respect to $x$ and $f_{y x}^{\prime \prime}$ is, for each $x$, a derivative function with respect to $y$.

I am unable to prove or to contradict this conjecture, but I can establish the following:

TheOREM. Let $f$ be a real function defined in the plane domain $G$. Suppose that $f_{x y}^{\prime \prime}$ and $f_{y x}^{\prime \prime}$ exist and are finite in $G$ and $f_{x}^{\prime}$ is continuous with respect to $x$. Then $f_{x y}^{\prime \prime}=f_{y x}^{\prime \prime}$ in $G$ if and only if $f_{y x}^{\prime \prime}$ admits, for each $x$, a primitive function with respect to $y$ and this primitive is, for each $y$, continuous with respect to $x$.

PRoof. Let $f_{x y}^{\prime \prime}=f_{y x}^{\prime \prime}$ in $G$. It follows immediately that $f_{y x}^{\prime \prime}$ admits $f_{x}^{\prime}$ as a primitive function with respect to $y$. But by hypothesis $f_{x}^{\prime}$ is continuous with respect to $x$.

To prove the sufficiency, we remark first that $f_{y x}^{\prime \prime}$, considered as a function of $x$, is a finite derivative function, therefore it is integrable with respect to $x$ in the Denjoy-Perron sense. Without loss of generality, we may assume that $G$ is a rectangle: $a \leqq x \leqq a+h, b \leqq y \leqq b+k$. Put

Received by the editors January 4, 1960 and, in revised form, June 17, 1960. 


$$
A(x, y)=\int_{a}^{x} f_{y t}^{\prime \prime}(t, y) d t
$$

the integral being taken here as everywhere in this paper in the Denjoy-Perron sense. From (1) it follows that $A(x, y)$ is, for each $x$, a derivative function with respect to $y$, hence integrable in the Denjoy-Perron sense. Put

$$
B(x, y)=\int_{b}^{y} A(x, u) d u
$$

We have

$$
B(x, y)=f(x, y)-f(a, y)-f(x, b)+f(a, b) .
$$

Denote by $\Delta x$ the increase of $x$ and by $\Delta B$ the corresponding increase of $B(x, y)$.

By the Lagrange formula, we get

$$
\frac{\Delta B}{\Delta x}=\int_{b}^{y} f_{u x}^{\prime \prime}(x+\theta \Delta x, u) d u
$$

$\theta$ being a function of $y$ and $u$ and such that $0<\theta<1$.

We shall show that, for $\Delta x \rightarrow 0$, the integral of the second member of (4) tends to

$$
\int_{b}^{y} f_{u x}^{\prime \prime}(x, u) d u .
$$

Really, $f_{u x}^{\prime \prime}$ is, by hypothesis, a derivative function with respect to $u$. Therefore there is a function $\phi(x, u)$ such that

$$
\frac{\partial \phi}{\partial u}=f_{u x}^{\prime \prime} .
$$

But $\phi(x, u)$ is, by hypothesis, continuous with respect to $x$, hence, for a sequence $\omega_{n} \rightarrow 0$, we get

$$
\lim _{n \rightarrow \infty} \phi\left(x+\theta_{n} \omega_{n}, y\right)=\phi(x, y), \quad \lim _{n \rightarrow \infty} \phi\left(x+\theta_{n} \omega_{n}, b\right)=\phi(x, b)
$$

where, by $\theta_{n}$ we have denoted the value of $\theta$ corresponding to the value $\omega_{n}$ of $\Delta x$. Therefore

$$
\lim _{n \rightarrow \infty} \int_{b}^{y} f_{u x}^{\prime \prime}\left(x+\theta_{n} \omega_{n}, u\right) d u=\int_{b}^{y} f_{u x}^{\prime \prime}(x, u) d u .
$$

From (4) and (5) it follows 


$$
\frac{\partial B}{\partial x}=\int_{b}^{y} f_{u x}^{\prime \prime}(x, u) d u .
$$

On the other hand, from (3) we get

$$
\frac{\partial B}{\partial x}=f_{x}^{\prime}(x, y)-f_{x}^{\prime}(x, b) \text {. }
$$

From (6) and (7) it follows

$$
f_{x y}^{\prime \prime}(x, y)=\frac{\partial}{\partial y}\left[\int_{b}^{y} f_{u x}^{\prime \prime}(x, u) d u\right] .
$$

But since $f_{u x}^{\prime \prime}(x, u)$ is, by hypothesis, a finite derivative function with respect to $u$ and since the indefinite Denjoy-Perron integral of a finite derivative function furnishes the primitive function, we get

$$
\frac{\partial}{\partial y}\left[\int_{b}^{y} f_{u x}^{\prime \prime}(x, u) d u\right]=f_{y x}^{\prime \prime}(x, y)
$$

therefore, combining (8) and (9), we obtain

$$
f_{x y}^{\prime \prime}(x, y)=f_{y x}^{\prime \prime}(x, y)
$$

and the theorem is completely proved.

REMARK 1. The continuity of $f_{x}^{\prime}$ with respect to $x$ can be replaced by the continuity of $f_{x}^{\prime}$ with respect to each sequence by which $f_{y x}^{\prime \prime}$ is continuous with respect to $x$.

REMARK 2. The necessary and sufficient condition given by the above theorem holds particularly in the case that all second-order partial derivatives exist and are finite in $G$. On the other hand, it is to be remarked that for the commutativity almost everywhere of the second-order cross partial derivatives it suffices that the first-order partial derivatives exist in $G$ and the second-order partial derivatives exist almost everywhere in $G[1]$.

REMARK 3. There exists a function whose first-order partial derivatives are continuous in $G$ and whose second-order cross partial derivatives exist in $G$ but are different on a set of positive measure [3].

\section{REFERENCES}

1. A. E. Currier, Proof of the fundamental theorems on second-order cross partial derivatives, Trans. Amer. Math. Soc. vol. 35 (1933) pp. 245-253.

2. J. Geffroy, Sur une relation entre les dérivées partielles $f_{x y}^{\prime \prime}$ et $f_{y x}^{\prime \prime}$, Acad. Roy. Belg. Bull. Cl. Sci. 5e Série, vol. 42 (1956) pp. 533-542.

3. G. P. Tolstov, On second-order partial derivatives, Mat. Sb. vol. 24 (1949) pp. 27-51 (in Russian).

Institute of Mathematics, R. P. R. Academy, Bucharest, Rumania 